\title{
Study of unstable fracture of Distal Tibia and its outcome managed with Minimally Invasive Plate Osteosynthesis (MIPO)
}

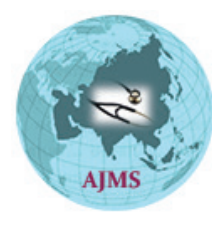

\author{
Pratyenta Raj Onta', Niraj Ranjeet ${ }^{2}$, Krishna Wahegaonkar ${ }^{3}$, Krishna Sapkota $^{2}$, \\ Pabin Thapa ${ }^{1}$, Upendra Jung Thapa ${ }^{1}$ \\ ${ }^{1}$ Lecturer, ${ }^{2}$ Assistant Professor, ${ }^{3}$ Associate Professor Department of Orthopedics, Manipal Teaching Hospital, Phulbari, \\ Pokhara, Nepal
}

Background: Distal tibia fracture is one of the difficult fractures to manage. There are many treatment options from conservative management to operative management. Each treatment methods have its advantages and disadvantages. Minimally invasive percutaneous osteosynthesis (MIPO) in distal tibia fracture has better outcome due to the advantages of minimal soft tissue dissection, preservation of periosteum and fracture hematoma. There is indirect reduction of fracture with correction of axial and rotation malalingement. Aims and Objective: The aim of the current study was to evaluate the functional and clinical outcome of distal tibia fracture treated with distal tibia anatomical contoured plate by MIPO technique. Materials and Methods: This was a prospective study that included 56 patients of which 37 were males. 31 patients had right sided distal tibia fracture. The most common mechanisms of injuries were road traffic accidents and fall injury. There was 6 cases of type I open fracture and 3 cases of type II open fracture. The patients were followed up at 2 weeks, 6 weeks, 3 months, 6 month and 12 months' time. Result: The mean age of the patients was 45.89 years. The mean time for partial and full weight bearing was 7.29 weeks and 14.29 weeks respectively. Radiological union was seen at 17.75 weeks. There was no case of nonunion or delayed union. The most common complication was ankle stiffness (5/56). Conclusion: MIPO is a good treatment option for unstable distal tibia fracture because of minimal soft tissue dissection and preservation of periosteum and fracture hematoma.
Access this article online Website:

http://nepjol.info/index.php/AJMS DOI: 10.3126/ajms.v9i5.20330 E-ISSN: 2091-0576 P-ISSN: 2467-9100

Key words: Distal tibia fracture; MIPO; Plate fixation

\section{INTRODUCTION}

The incidence of fracture of distal tibia is $0.6 \%$ and it constitutes about $10-15 \%$ of all tibia fracture cases. ${ }^{1}$ The incidence is on the rise due to the increase in the incidence of motor vehicle accidents and longer life expectancy. There are many methods for management of unstable distal tibia fractures. Conservative management in distal tibia fracture is usually done in younger age group and when the fracture is stable with minimal shortening but this treatment method has complications like long time immobilization leading to joint stiffness and disuse osteoporosis, malunion and limb length discrepancy. ${ }^{2-4}$ Open reduction and plate fixation though has better fracture reduction, better fixation and early mobilization, needs extensive soft tissue dissection and also has higher rate of complications like infection, delayed union due to drainage of fracture hematoma, non-union and big scar marks. ${ }^{5,6}$ External fixators and ring fixators can be used in cases when there is open fracture and severe intraarticular comminution but these methods are also not free from complications like pin track infection, cumbersome framing and non-union and malunion. ${ }^{7}$

Nailing is always a preferred method of treatment in fracture shaft of tibia but it becomes technically difficult in cases when the fracture is in the distal third as the 
fracture line may extend to tibial pilon., ${ }^{8,9}$ Though nailing has advantages of preservation of fracture hematoma and avoiding damage to the overlying skin, the angular and rotational stability is not achieved in distal tibia due to the widening of the metaphysis in distal tibia and difficulty to fix the nail with two distal screws. ${ }^{10,11}$

Minimal invasive plate osteosynthesis (MIPO) using the anatomical contoured distal tibial plate has benefits of minimal soft tissue damage, minimal periosteum elevation, preservation of fracture hematoma, stable fixation and early mobilization. ${ }^{12} \mathrm{MIPO}$ is done without periosteum elevation and opening of the fracture site. MIPO aims to correct rotational and axial malalingement and also aims to achieve limb length. Fracture healing in MIPO occurs by formation of callus. ${ }^{13}$ The aim of our study was to evaluate the functional and clinical outcome of distal tibia fracture treated with distal tibia anatomical contoured plate by MIPO technique.

\section{MATERIALS AND METHODS}

This was a prospective study conducted from January 2015 to January 2018in department of Orthopedics, Manipal Teaching Hospital, Pokhara, Nepal after getting the ethical clearance from the IRC. The inclusion and exclusion criteria for the study were as follows.

\section{Inclusion criteria}

1. Distal tibia fracture with or without simple intra articular extension.

2. Type I and II open fracture.

3. Age more than 18 years.

4. Case of poly trauma with minor head injury not requiring neurosurgery intervention.

5. Duration of injury less than 2 weeks.

\section{Exclusion criteria}

1. Open fracture more than typeII.

2. Poor local skin condition.

3. Pathological fracture.

4. Associated neurological or vascular injury.

5. Fracture in pediatric age group.

6. AO type C3 (articular comminution).

7. Patient with compartment or impending compartment syndrome.

All the patients with distal tibia fracture who were planned for surgery were evaluated and above knee posterior slab was given. If there were swelling in and around the ankle joint the surgery was delayed till the swelling subsided and there was wrinkle sign. Open fractures were treated with intravenous antibiotics and lavage prior to surgery. All patients were classified according to AO classification system. They underwent pre anaesthesia checkup and clearance was obtained before the surgery.

\section{Operative procedure}

After administration of spinal or general anesthesia the patients were placed supine on a radiolucent table. Tourniquet was used in all patients. The part was painted with $10 \%$ povidone iodine solution and draped. Small incision (size about 2.5 to $5 \mathrm{~cm}$ ) was given over the medial malleolus. Saphenous nerve and vein were isolated and retracted. Pre contoured distal tibia locking plate was inserted from the medial malleolus. The position of plate was checked in image intensifier. The fracture was reduced with manual traction and manipulation. The plate was fixed with at least 3-4 locking screws proximally and distally (preferably 4 locking screws proximally and distally). Fibular plating was done with narrow DCP for achievement of accurate leg length whenever it was necessary.

Results were compiled. Statistical analysis was done usingSPSS 16.

\section{Postoperative}

Postoperatively all patients were given below knee posterior slab for 4-6 weeks. Wound dressing was done on every third day. Sutures were removed after 14 days. Active and passive movements of knee and ankle were encouraged. Partial weight bearing was started after 6 to 8 weeks' time and gradually increased to full weight bearing. The patients were followed up at 2 weeks, 6 weeks, 3 months, 6 months and 12 months' interval. Fracture was considered to be healed when the patient had radiological callus bridging the fracture and clinically when the patient had no pain on full weight bearing.

\section{RESULTS}

There were total 56 patients included in the study among whom $37(66.1 \%)$ were males. The mean age of the patients was 45.89 years (range 21-78 years) with standard deviation of 16.36. Right side distal tibia fracture was found in $31(55.4 \%)$ cases. In this study 47 patients $(83.9 \%)$ had closed fracture, 6 patients $(10.7 \%)$ had type I open fracture and 3 patients (5.4\%) had type II open fracture. The mechanism of injury in 25 patients $(44.6 \%)$ was road traffic accident followed by fall injury in 21 patients $(37.5 \%)$. The cause of fracture in distal tibia fracture due to sports injury and twisting injury was $6(10.7 \%)$ and $4(7.1 \%)$ respectively. Fibula plating was done in 8 patients $(14.3 \%)$ as shown in Table I.

The mean time for partial weight bearing was 7.29 weeks (6-10 weeks) with standard deviation of 0.929 whereas 


\begin{tabular}{lll}
\multicolumn{2}{l}{ Table I: Demographic data } & \\
\hline S.no & Demography & No. \\
\hline 1. & Age (years) & Mean age 45.89 years (21-78 years) \\
2. & Sex (Male/Female) & $37 / 19$ \\
3. & Mode of Injury (RTA/Fall/Twisting/Sport) & $25 / 21 / 4 / 6$ \\
4. & Affected limb (Right/Left) & $31 / 25$ \\
5. & Closed fracture & 47 \\
& Type I open fracture & 6 \\
6. & Type II open fracture & 3 \\
& AO classification & 20 \\
& $43 A 1$ & 15 \\
& $43 A 2$ & 10 \\
7. & $43 A 3$ & 11 \\
8. & $43 B 1$ & 8 \\
& Fibular plating & 7.29 weeks $(6-10$ weeks) \\
9. & Weight bearing (weeks ) & 14.29 weeks (12-20 weeks) \\
& Partial & 17.75 weeks (16-24 weeks) \\
\hline
\end{tabular}

the mean time for full weight bearing was 14.29 weeks (12-20 weeks) with standard deviation of 2.40. Radiological union in our study was seen in mean time period of 17.75 weeks (16-24 weeks) with standard deviation of 1.46 . Ankle stiffness was seen in 5 patients $(8.9 \%)$.Hardware prominence was seen in 3 patients $(5.4 \%)$. Exposure of fibular plate was seen in one patient who underwent early removal of fibular plate. One patient with type I open fracture had superficial wound infection which was treated adequately with intravenous antibiotics and regular wound dressing.

\section{DISCUSSION}

Distal tibia fracture with or without articular extension is one of the difficult fractures to treat due to its proximity to ankle joint, significant soft tissue injury and comminution due to trauma. The long term outcome of the fracture is determined by the mechanism of injury, soft tissue condition, extent of comminution and articular damage at the time of injury. The goal of surgical management for distal tibia fracture is anatomical reduction, mechanical stability with prevention of rotation and angulation and early mobilization.

MIPO technique has the benefit of indirection reduction of fracture with percutaneous plate fixation minimizing the soft tissue dissection and preservation of fracture hematoma. ${ }^{14}$ Due to the high success rate and good clinical outcome MIPO has become good treatment option for fracture distal tibia. ${ }^{15,16}$

The study conducted by Hasenboehler et al. found that MIPO when used as bridging plate prolongs the union of the fracture according to the fracture pattern. ${ }^{17}$ In our study we did not find any cases of nonunion or delayed union.
All fractures had union irrespective of the fracture pattern. The average time for radiological union was 17.75 weeks which was similar to the study done by Guo J.J et al. (17.6 weeks), Hazarika S et al. (18 weeks), Senthilkumar M et al. (18weeks), Kumar VK et al. (16.1weeks), Lau et al. (18.7weeks) and Gupta RK et al. (19 weeks). ${ }^{18-23}$

As compared to intramedullary nailing the rate of wound infection in conventional plating depends on the condition of soft tissue at the time of injury, the effect of which in MIPO has to be compared. ${ }^{9}$ The reported rate of wound infection is $2.6 \%$ to $14.6 \%$ and it depends on the inclusion or exclusion of open fracture. In our study we included type I and II open fractures. There were 6 type I and 3 type II open fractures and we found that the union time was late in open fractures (mean 18.5 weeks in type I and 20.66 weeks in type II). The reason for late union may be due to drainage of fracture hematoma. Superficial wound infection was seen in one case in type I open fracture which was managed with Intravenous antibiotics and regular dressing of the wound.Saphenous nerve and vein injuries have been reported in studies but we did not have any case with saphenous nerve or vein injury in our study. ${ }^{24,25}$

\section{CONCLUSION}

MIPO for unstable distal tibia fracture is a good treatment option with good results with few complications. Due to the limitation of soft tissue dissection and preservation of fracture hematoma there is early fracture union resulting into early pain free mobilization and early return to work.

\section{REFERENCES}

1. Bucholz R, Court-Brown C and Rockwood C. Rockwood and Green's Fractures in adults. New York: Lippincott; 2015: 98 
2. Ruedi TP and Allgower M. The operative treatment of intra articular fractures of the lower end of tibia. ClinOrthop1979; 138:105-110.

3. Walker C, Garg A and McQueen MM. Half-ring external fixation in the management of tibial plafond fractures. Journal of orthopaedic trauma 1999;13(3):200-206.

4. Bedi A, Le TT and Karunakar MA. Surgical treatment of nonarticular distal tibia fractures. JAAOS-Journal of the American Academy of Orthopaedic Surgeons. 2006;14(7):406-416.

5. Teeny SM and Wiss DA. Open reduction and internal fixation of tibial plafond fractures. Variables contributing to poor results and complications. Clinical orthopaedics and related research 1993; (292):108-117.

6. Olerud $S$ and Karlström $G$. Tibial fractures treated by $A O$ compression osteosynthesis: experiences from a five year material. ActaOrthopaedicaScandinavica. 1972; 43:1-4

7. Rammelt S, Endres T, Grass R and Zwipp H. The role of external fixation in acute ankle trauma. Foot and ankle clinics 2004 9(3):455-474.

8. Ronga M, Longo UG and Maffulli N. Minimally invasive locked plating of distal tibia fractures is safe and effective. Clinical Orthopaedics and Related Research2010; 468(4):975-982.

9. Im GI and Tae SK. Distal metaphyseal fractures of tibia: a prospective randomized trial of closed reduction and intramedullary nail versus open reduction and plate and screws fixation. Journal of Trauma and Acute Care Surgery 2005; 59(5):1219-1223.

10. Fan CY, Chiang CC, Chuang TY, Chiu FY and Chen TH. Interlocking nails for displaced metaphyseal fractures of the distal tibia. Injury. 2005; 36(5):669-674.

11. Mosheiff R, Safran $O$, Segal $D$ and Liebergall $M$. The unreamedtibial nail in the treatment of distal metaphyseal fractures. Injury1999; 30(2):83-90.

12. Farouk $\mathrm{O}$, Krettek $\mathrm{C}$, Miclau $\mathrm{T}$, Schandelmaier $\mathrm{P}$, Guy $\mathrm{P}$ and Tscherne $\mathrm{H}$. Minimally invasive plate osteosynthesis and vascularity: preliminary results of a cadaver injection study. Injury1997; 28:A7-A12.

13. Krackhardt $\mathrm{T}$, Dilger $\mathrm{J}$, Flesch $\mathrm{I}$, Höntzsch D, Eingartner $\mathrm{C}$ andWeise K. Fractures of the distal tibia treated with closed reduction and minimally invasive plating. Archives of orthopaedic and trauma surgery 2005; 125(2):87-94.

14. McFerran MA, Smith SW, Boulas HJ and Schwartz HS
Complications encountered in the treatment of pilon fractures. Journal of orthopaedic trauma1992; 6(2):195-200

15. Borg T, Larsson S and Lindsjö U. Percutaneous plating of distal tibial fractures: preliminary results in 21 patients. Injury 2004; 35(6):608-614

16. Ghera S, Santori FS, Calderaro M and Giorgini TL. Minimally invasive plate osteosynthesis in distal tibial fractures: pitfalls and surgical guidelines. Orthopedics2004; 27(9):903-905.

17. Hasenboehler E, Rikli D and Babst R. Locking compression plate with minimally invasive plate osteosynthesis in diaphyseal and distal tibial fracture: a retrospective study of 32 patients. Injury2007; 38(3):365-370.

18. Guo JJ, Tang N, Yang HL and Tang TS. A prospective, randomised trial comparing closed intramedullary nailing with percutaneous plating in the treatment of distal metaphyseal fractures of the tibia. NederlandsTijdschriftvoor Traumatologie2010; 18(6): 185-186

19. Hazarika S, Chakravarthy $\mathrm{J}$ and Cooper J. Minimally invasive locking plate osteosynthesis for fractures of the distal tibiaresults in 20 patients. Injury2006; 37(9):877-887.

20. Senthilkumar M, Vanaj $P$, Sujith $H$ and Anandan $H$. Distal Tibial Fractures Managed with Locking Compression Plate using Minimally Invasive Plate Osteosynthesis Technique: A Case Study. International journal of scientific study 2016; 4(8):43-47.

21. Kumar VK, Sherafudeen $Y$ and Muhammed F. Role of minimally invasive percutaneous plate osteosynthesis in the treatment of distal diametaphysealtibial fractures. Asian Pac. J.HealthSci 2014; 1(4S):14-19.

22. Lau TW, Leung F, Chan CF and Chow SP. Wound complication of minimally invasive plate osteosynthesis in distal tibia fractures. International orthopaedics 2008, 32(5):697-703.

23. Gupta RK, Rohilla RK, Sangwan K, Singh V and Walia S. Locking plate fixation in distal metaphyseal tibial fractures: series of 79 patients. International orthopaedics. 2010; 34(8):1285-1290.

24. Cheng $\mathrm{W}, \mathrm{Li} Y$ and Manyi W. Comparison study of two surgical options for distal tibia fracture-minimally invasive plate osteosynthesis vs. open reduction and internal fixation. International orthopaedics 2011; 35(5):737-742.

25. Ozsoy MH, Tuccar E, Demiryurek D, Bayramoglu A, Hayran M, Cavusoglu AT, et al. Minimally invasive plating of the distal tibia: do we really sacrifice saphenous vein and nerve? A cadaver study. Journal of orthopaedic trauma. 2009; 23(2):132-138.

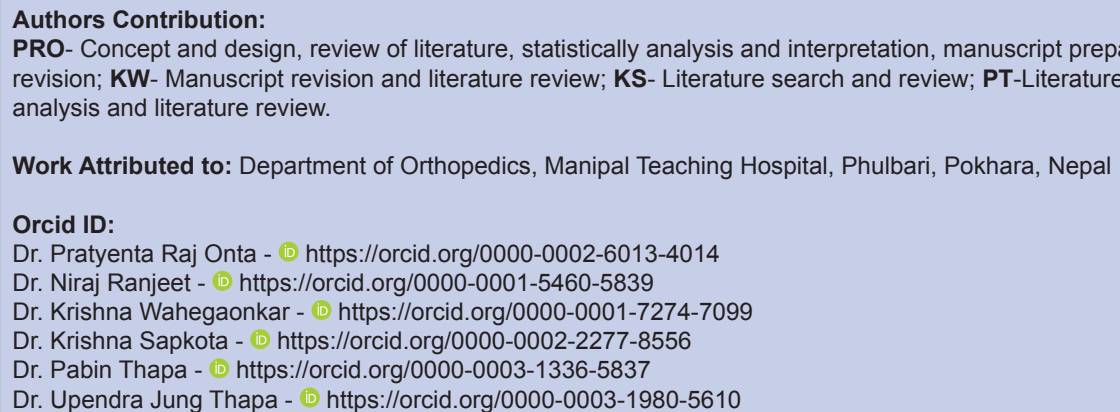

